\title{
FACTORS THAT FACILITATE ORGANISATIONAL CHANGE IN COMPLEX SYSTEMS
}

\author{
P.M. DICKENS \\ Tyndale University College, Canada.
}

\begin{abstract}
This paper explores the capacity of complex systems to find their own form of order and coherence, often referred to in terms of self-organisation emergent change, then asking the question, 'What can organisational leaders do to create the systems and structures that would facilitate emergent change?' Emergent change comes from within and through the active members of a system and is not imposed according to some external prompting or design. This results in the sort of change capacity that enables an organisation to be agile and resilient in highly volatile times. I have identified seven key organisation-specific factors that facilitate emergent change. These include: executive engagement, purposeful orientation, a culture of experimentation, a safe-fail culture, collaborative decision-making, collaborative quality measures, and intentional learning. These factors were initially identified through an extensive literature review, interviews with the CEOs of 15 Canadian healthcare organisations, and a think tank of subject matter experts. This resulted in the use of exploratory factor analysis to validate a survey that can be used to assess the presence or absence of these factors in a specific organisation, thus providing leaders with a framework for change.

Keywords: change, collaboration, creativity, emergence, leadership, learning, self-organisation.
\end{abstract}

\section{THE CHALLENGE}

As organisational life becomes more and more complex, today's dominant management paradigms no longer seem to suffice. The problem is that while many leaders readily acknowledge the challenges and limitations of traditional approaches, they have a limited range of options for dealing with a highly complex world. This paper identifies new ways of thinking about and responding to complexity: not by trying to simplify it, but by embracing the inherent capacity of complex systems to find their own form of order and coherence.

The level of complexity and unpredictable change faced by organisations today is unprecedented. Globalisation, rapidly changing technologies, unpredictable geopolitical environments, and increasingly informed consumers bring tremendous challenges for organisations trying to stay afloat in such 'permanent white water' [1]. Eoyang and Holladay [2] suggest that we can best understand this complexity along three continua: bounded and unbounded systems, few factors and many factors, and one-way and two-way causality. When the problems we face are relatively simple, the system within which we operate is described as bounded or contained. We focus on the implications of a decision on our organisation or department. A problem becomes more complicated when there are multiple systems impacted by any one decision, and a problem is truly complex [3] when we realise there are almost innumerable systems impacted by a change (Zimmerman et al. [3] provide a useful series of metaphors to understand the difference between simple, complicated, and complex. Baking a cake is simple: the recipe is known, tested, and replicable. Launching a rocket is complicated: multiple formulae, careful attention to sequence, but ultimately replicable. Raising children is complex: formulae are of limited value, no two are alike, and the results are ultimately unpredictable). Similarly, the exponential increase of factors impacting a single decision moves the marker along the second continuum and the presence of multiple causalities makes more and more challenges extremely complex. Little wonder that Eoyang suggests that, 'well-informed trial and error is the only viable strategy' $[2$, p. 20] in today's highly complex world. Hence, I see an urgent need to 
develop a better understanding of complexity and complex, adaptive systems (CAS) from a leadership perspective.

\section{BROAD DEFINITIONS OF CAS}

The origins of complexity theory lie in the study of deterministic systems such as biological units, numbers, and subatomic particles - in other words, systems that lack free will and choice. However, the reality for leaders is that theirs are human-based systems and, as such, are subject to the inherent complexities of both the human body and, perhaps more challenging, the human mind and will. There are multiple definitions of CAS. According to Plowman et al. [4], some of the characteristics of CAS include: (1) they are made up of many agents who act and interact with each other in unpredictable ways; (2) they are sensitive to initial conditions; (3) they adjust their behaviour in the aggregate in unpredictable ways; (4) they oscillate between stability and instability; and (5) they produce emergent actions when approaching disequilibrium [4].

These descriptors of various attributes of CAS are central to the factors that this paper identifies as key to facilitating emergent change. What this paper tries to do is to take the discussion of attributes further by trying to assess the presence or absence of such factors, in order to provide leaders with suggested strategies to enhance their organisational capacity for change.

In a complex, adaptive social system, no individual agent, or designated group of agents, determines the local interaction principles of others and there is no centralised direction managing the patterns of behaviour of the system as a whole or of the evolution of those patterns. The evolution is, in fact, referred to as self-organisation. It is important to recognise that conceptually, emergent change is neither positive nor negative: it simply is. A riot is as much an example of self-organisation and emergent change as was the gathering that heard Martin Luther King's famous 'I have a dream' speech. It is my belief that we can distinguish positive emergent change in a social system because positive emergent change contributes to the mutual purposes of both leaders and followers who share common values and intend real change [5].

Many researchers have suggested that self-organisation is the key concept to be drawn from complexity theory.

Self-organisation is a characteristic of all social systems that operates whether we recognise it or not. By recognising it, however, we can begin to influence it to facilitate better outcomes. Self-organisation is the process by which people mutually adjust their behaviours in ways needed to cope with changing internal and external environmental demands [3, 6-9].

Self-organisation is a process whereby the coherence of a system spontaneously increases, without this increase being controlled by formal directive or an external system. This spontaneous increase in order is a construct that would stand in marked contrast to the concept of entropy described in classic Newtonian physics. Some would argue that there is enormous benefit to organisations that can develop the capacity for self-organisation. As Lindberg et al. suggest, 'The more self-organised the change (in an organisation), the higher the whole system's performance will be' [10]. That being said, it does not provide a guarantee. In the same way, certain parenting approaches increase the likelihood of a well-adapted child, there are a great many other intervening variables that may prevent this outcome from happening.

\section{RESEARCH METHODS}

The research was based on a review of the literature, interviews with CEOs in 15 large, complex health care organisations, feedback from a panel of subject matter experts, and my own organisation 
development practice experience. This allowed me to identify nine initial constructs of interest, which were then integrated into the initial Organisational Resilience Assessment (ORA). One hundred and sixty-two senior leaders, managers, and staff at a hospital in Toronto, Canada completed the survey. I then completed a principal component analysis of the constructs to validate the survey. From this analysis, seven valid factors emerged. I assessed the link between the factors and organisational performance by conducting two focus groups at the study site. The focus groups confirmed the correlation between the factors and key organisational performance indicators. The factors include: purposeful orientation, a high level of executive engagement, a safe-fail culture, collaborative decision-making processes, collaborative quality processes, intentional learning process, and culture of experimentation.

\subsection{Initial interviews}

Prior to the development of the ORA survey, I interviewed senior leaders in the Ontario healthcare sector. This included 13 hospital CEOs, a Deputy Assistant Minister in the Ministry of Health and Long-Term Care, and a senior researcher. There was broad consensus that the system is extremely complex and that the complexity is increased by the lack of clarity regarding the role of the Local Health Integration Networks (LHINs), Ontario's initial foray into some form of regional delivery model. Broadly speaking, all the interviewees pointed to the importance of several of the elements of the seven validated factors that emerged from the exploratory factor analysis, particularly the importance of leadership engagement and organisation-wide leadership development in order to provide the skills to engage in the sorts of collaborative behaviours indicated by the survey results. The feedback from the interviews aligned with the information drawn from the literature review thus forming the basics of the initial constructs of interest for the survey.

\subsection{Development and evaluation of the ORA survey}

The survey was developed following a careful analysis of the literature and the interviews, looking for common patterns and themes. From this analysis, seven initial constructs of interest were developed. A panel of individuals with some experience with emergent change was then asked to identify specific factors that they felt had facilitated the change. From this feedback, two new constructs emerged, as well as the language that began to frame items within each construct. A draft of the survey was then circulated to the panel for further refinement. Once the draft had gone through two more iterations, an Ethics Review was completed at the study site. The site was selected because it consistently achieved strong financial, patient care, and staff satisfaction results.

The survey was conducted using Survey Monkey ${ }^{\circledR}$ and allowed for both quantitative and qualitative responses. One hundred and seventy-four leaders and staff at the hospital responded to the survey, but 10 did not complete it, leaving $\mathrm{N}=164$. The survey group was intentionally limited to past participants in a formal leadership development programme at the hospital, in the expectation that it would increase the response rate. It did (response rate was 40\%), but it also appears to have skewed the results, which were consistently above the mean. Since this was the first use of the survey, there was not a pool of data against which to assess the results; but since the intent was validation of the survey rather than the formal assessment of the hospital, the process served its purpose. Quantitative data, in the form of individual comments on each of the factors, was significantly more balanced, but the overall tone of responses indicated affirmation of all seven factors.

The results were then loaded into SPSS ${ }^{\circledR}$, a software package that facilitates an exploratory factor analysis. After careful analysis, five factors with four to six items in each factor indicated Cronbach's 
alpha over 0.700 , which is indicative of their internal validity. Two more emerged with alpha between 0.550 and 0.700 , which indicated some level of validity, but also indicated the need for further refinement. A second version of the survey was then prepared.

\section{THE SEVEN FACTORS THAT FACILITATE EMERGENT CHANGE}

This study undertook the task of understanding and validating organisational or strategic factors that might facilitate positive emergent change. What emerged from the results of the study were as follows:

\subsection{Executive engagement}

The degree to which senior management demonstrates support for and commitment to nonhierarchical approaches. This had a very strong internal validity and aligned with the input of the interviews. It was later validated by the focus groups. The importance of commitment and support from senior leadership is almost counterintuitive when thinking in terms of CAS, where the emphasis tends to focus on a more distributed model of leadership [11]; however, a previous research at the study site hospital indicated the significance of senior-level support [13]. As one interviewee in that study said, 'I know it sounds a bit silly, but it was almost as if we needed, or wanted, mom and dad's permission to experiment'. This factor includes the level of visibility and approachability of the $\mathrm{CEO}$ and members of the senior executive team, and the degree to which they enable staff to fully engage in, and take ownership for, creative problem-solving.

The need for an intentional and widespread leadership development strategy was implicit in the interviews when the topic of executive engagement was discussed. The implication was that, without a commitment to distributed leadership, senior leaders were inevitably drawn into a more managerial role, which limited their ability to engage relations across the organisation. Equipping leaders at all levels of the organisation was viewed as central to developing the innovative and decision-making capacity of the organisation and supported a commitment to ongoing, non-clinical/technical learning.

\subsection{Safe-fail culture}

The degree to which the organisation is perceived to be comfortable with making mistakes, learning, and moving on without fear of recrimination. The idea that organisations need to create safe spaces to foster innovation has been present since organisations began to talk about innovative cultures [14]. However, historically, these safe spaces have been segregated and given terms like skunk works. Today, it would appear that the pace of change facing every function of an organisation does not often allow for the luxury of such separateness. Instead, organisations need to create and support safe holding spaces for experimentation [15]. While the survey validated the importance of this, written feedback and the focus groups indicated that, while vital, it was not universal at the study site. It appears to be contingent on the level of trust between staff and management.

\subsection{Collaborative decision processes}

The degree to which people throughout the organisation have timely input into the decisions that affect the work that they do. Holman et al. suggested that 'what keeps (a) system whole over time is a commitment to collaborative meaning making' [16]. The opportunities for such collaboration can be left to chance, but the strategic organisation takes the lead in creating collaborative work structures. Tekell et al. (in Holman et al. [16]) described collaborative work systems arising from 'a holistic 
design process that creates the framework for successfully changing the organisation to support collaboration and improve business results' [16, p. 437]. These collaborative structures create the space within which people at all levels of the organisations can discuss the implications of the three continua discussed earlier and engage in their own adaptive action. The movement to interprofessional education and collaborative care models in health care is evidence of this shift. The study site has committed significant time to developing collaborative care models, so there was intentionality about this factor that was indicated by the high variance from the mean exhibited in the survey results.

\subsection{Collaborative quality}

The degree to which decisions about quality measures and strategies are defined by the people doing the work, supported with the data they need to make decisions. The primary rationale for the inclusion of this factor comes from Wergin's study [17] in which he reported on the findings of an extensive Pew Charitable Trust study on quality. Wergin intended to identify the necessary conditions for quality in a post-secondary education environment. What he found was that it was key that quality occurred at the departmental level and with academic chairs, rather than at the most senior levels. The same is true in health care organisations: people closest to the patient have the best understanding of the necessary quality metrics.

While Wergin found that there were six necessary conditions, they were not sufficient. One other factor differentiated the really high-quality departments: evaluation policies were flexible and decentralised. Each department defined what quality meant in their context and then were held accountable for meeting that standard. 'The only institution-wide requirement was that departments include in their study an analysis of how they contribute to the mission of the institution' [17, p. 35]. Wergin found that six necessary conditions have to exist before there is sufficient trust to embrace decentralised evaluation. Based on Wergin's conclusions, a localised quality framework was deemed a distinct factor in this study. During the focus groups, several people pointed out that this approach to quality was central to the Lean Quality Improvement approaches that are a systemic part of the hospital; so, the importance of this factor was not a surprise to the participants.

\subsection{Intentional learning processes}

The degree to which there is both formal and informal support for both technical and relational skills and the willingness of the organisation to learn as it goes. Learning and constant adaptation are central elements of a CAS's capacity to adapt and change [18].

Even the simplest adaptive system has some purpose, namely, to perform some task. It follows that, unlike agents in deterministic systems, agents in all adaptive systems adjust their behaviour in light of its consequence for their purpose. In other words, adaptive systems learn, at the very least, in a simple single-loop manner, whereas a deterministic system does not. A great deal of attention is rightly placed on the capacity of the individual agents to learn and adapt [19] and for those same agents to engage in opportunities for shared learning [20,21]; however, this study is focused on the organisational factors that contribute to emergence, so the focus here is on system structures. From that perspective, the question becomes whether or not the agents of the system are intentionally aligned to teams and groups that will facilitate learning. Several of the individual comments in the survey spoke of the value that respondents attached to the degree of commitment the organisation has made to their personal and professional development in terms of access to formal courses and workshops. However, there was also evident tension between availability and time: frontline staff, in particular, indicated that it was difficult for them to get away from their tasks to participate. 


\subsection{Culture of experimentation}

The degree to which people are willing to try new ideas and approaches, to listen to people who think differently than they do, and to welcome innovation. Central to this type of experimentation is having real-time access to meaningful data in order to constantly adapt and change. Like learning, feedback is a central concept in CAS theory. Complex systems are open systems with feedback loops that can enhance and stimulate positive emergence when there is a steady flow of real-time information, so that the agents within the system can adjust their innovative efforts in order to increase effectiveness. A system must not only receive, process, and retain information, but also respond to and produce some form of output or new data to which other elements of the system can then respond. The difference between the sorts of feedback mechanisms needed in complex adaptive organisations is that the organisation needs to be able to 'hold multiple and sometimes conflicting representations of environmental variety, retaining in their behavioural repertoire a range of responses, each of which operates at a lower level of specificity' [22]. Change in a CAS is encouraged by increasing information flow to all parts of the organisation and then pushing the authority to do something with that information as far out into the organisation as possible [6]. The importance of access to real-time data in order to adapt and improve was strongly evident in both the literature and feedback from the focus group.

\subsection{Purposeful orientation}

The degree to which people feel they have a common purpose and focus and share a passion for that purpose. Wheatley suggested that, 'We need to trust that something as simple as a clear core of values and vision, kept in motion by continuous dialogue, can lead to order' [23]. This begins to suggest a different way of thinking about an organisation's strategic framework or high-level organisational commitments. This framework creates the 'bounded instability' that allows for innovation and selforganisation [19]. Wheatley went on to suggest that, 'In human organisations, a clear sense of identity - of values, traditions, aspirations, competencies, and culture that guide the operation - is the real source of independence from the environment' [23, p. 94]. The mission of the study site is central to all decisions made in the study site, and several survey respondents commented on its visual prominence and intentionality in decision-making.

\section{IMPACT ON PERFORMANCE: A MOLECULAR METAPHOR}

Both the focus groups affirmed the importance of all seven factors to organisational performance, as measured by patient and staff satisfaction, financial performance, and quality outcomes. However, the groups were not able to draw a specific correlation between any one factor and any specific performance metric. This is consistent with the integrated nature of the factors. One of the participants likened the cluster of factors to a seven-atom molecule, in that you could remove one of the atoms and you would still likely have a 'something', but it would not be the original structure. This was a useful metaphor for the group. The groups also affirmed the transitory nature of each of the factors, suggesting the importance of leadership vigilance if the desired agility and resilience were to be sustained.

\section{CONCLUSIONS}

The interviews, literature review, and results of the initial survey are all congruent and, as one reviewer suggested, all seem to align with good leadership practices, which is certainly true. However, I would argue that what has been missing from the discussion about leadership practices in a 
complex environment has been a coherent, unifying theoretical framework, which I believe complexity theory provides. Further, the survey helps leaders and practitioners move complexity theory into something that is far more actionable.

It must be noted that this was an exploratory factor analysis, so caution needs to be shown when looking for effective ways to use the results of subsequent surveys. However, these seven factors have been validated statistically and their presence or absence can now be assessed through an online survey, available through the author. The results of such a survey may help to identify key points of leverage within an organisation. Survey results can then be linked to organisational performance through a series of focus groups, in order to develop new strategies and patterns of engagement.

What emerges from any discussion of factors facilitating emergence is that like the complex adaptive organisations they are intended to influence, no single factor stands alone, but rather it is the interdependence or combinatory nature [24, p. 147] of all of the factors that seems significant.

With this research, practitioners and organisational leaders can take steps to move beyond understanding complexity as a metaphor and begin to see it as a strategic framework within which they can begin to reshape their organisations. However, a final word of caution: this approach shapes and facilitates emergent change, but it is not a 'guarantee' of success. That is simply not in the nature of complexity.

\section{REFERENCES}

[1] Vaill, P.B., Learning as a way of being: Strategies for survival in a world of permanent white water. Jossey-Bass: San Francisco, CA, 1996.

[2] Eoyang, G.H. \& Holladay, R.J., Adaptive action: Leveraging uncertainty in your organization. Stanford University Press: Stanford, CA, 2013.

[3] Zimmerman et al., Edgeware; Insights from complexity science for health care leaders. VHA Inc: Irving, TX, 1998.

[4] Plowman, D.A., Solansky, S., Beck, T. E., Baker, L., Kulkarni, M. \& Travis, D.V. The role of leadership in emergent, self-organisation. Leadership Quarterly, 18(4), 341-356, 2007. doi: http://dx.doi.org/10.1016/j.leaqua.2007.04.004

[5] Rost, P. Leadership for the 21st Century. Praeger Publishing: Westport, CT, p. 102, 1993.

[6] Lichtenstein, B.B. \& Plowman, D.A., The leadership of emergence: A complex systems leadership theory of emergence at successive organisational levels. The Leadership Quarterly, 20(4), 617-630, 2009. doi: http://dx.doi.org/10.1016/j.leaqua.2009.04.006

[7] Arena, M.J. Understanding large group intervention processes: A complexity theory perspective. Organisation Development Journal, 27(1), 49-64, 2009.

[8] Lichtenstein, B.B., Emergence as a process of self-organising - new assumptions and insights from the study of non-linear dynamic systems. Journal of Organisational Change Management, 13(6), 526-544, 2000. doi: http://dx.doi.org/10.1108/09534810010378560

[9] Olson, E.E. \& Eoyang, G.H., Facilitating Organisation Change: Lessons from Complexity Science. Jossey-Bass: San Francisco, CA 2001.

[10] Lindberg, C., Nash, S. \& Lindberg, C. On the Edge: Nursing in the Age of Complexity. Plexus Press: Bordentown, NJ, 2008.

[11] Marion, R. \& Uhl-Bien, M. Introduction to the special issue on leadership and complexity. The Leadership Quarterly, 18(4), 293-296, 2007. doi: http://dx.doi.org/10.1016/j. leaqua.2007.04.001

[12] Uhl-Bien, M. \& Marion, R. Complexity Leadership. IAP: Charlotte, NC, 2008.

[13] Dickens, P., Cultural \& global dimensions of change: Power dynamics in a health care setting. Unpublished manuscript, Antioch University, Yellow Springs, OH, 2010. 
[14] Dombrowski, C., Kim, J.Y., Desouza, K.C., Braganza, A., Papagari, S., Baloh, P. \& Jha, S. Elements of innovative cultures. Knowledge and Process Management, 14(3), 190-202, 2007. doi: http://dx.doi.org/10.1002/kpm.279

[15] Heifetz, R., Grashow, A. \& Linsky, M., The practice of adaptive leadership: Tools and tactics for changing your organisation and the world. Harvard Business Press: Boston, MA, 2009.

[16] Holman, P., Devane, T. \& Cady, S. The Change Handbook: The Definitive Resource on Today's Best Methods for Engaging Whole Systems, 2nd edn., Berrett-Koehler: San Francisco, CA, p. 12, 2007.

[17] Wergin, J.F., Departments that Work: Building and Sustaining Cultures of Excellence in Academic Programs. Anker: Boston, MA, 2003.

[18] Westley, F., Patton, M.Q. \& Zimmerman, B., Getting to Maybe: How the World is Changed. Vintage Canada: Toronto, Canada, 2007.

[19] Stacey, R., Complexity and Creativity in Organisation. Berrett-Koehler: San Francisco, CA, p. 72, 1996.

[20] Argyris, C., On organisational learning. Blackwell: Cambridge, MA, 1992.

[21] Mesirow, J., Transformative Dimensions of Adult Learning. Jossey-Bass: San Francisco, CA, 1991.

[22] Boisot, M. \& Child, J. Organisations as adaptive systems in complex environments: The case in China. Organisational Science, 20(3), 237-252, p. 238, 1999.

[23] Wheatley, M., Leadership and the New Science. Berrett-Koehler: San Francisco, CA, 1994.

[24] Arthur, W.B., The Nature of Technology: What It is and How It Evolves. Free Press: New York, NY 2009. 